\title{
Reversible Ion Induced Modification of Consequent Secondary Electron Emission in Porous Silicon
}

\author{
G.D. Ruano ${ }^{1}$, J. Ferron ${ }^{1,2}$ and R.R. Koropecki ${ }^{*}, 1,2$ \\ ${ }^{I}$ INTEC, CONICET - UNL Güemes 3450 - 3000 Santa Fe, Argentina \\ ${ }^{2}$ Departamento de Ingeniería de Materiales. Fac. Ing. Química. UNL, Argentina
}

\begin{abstract}
We report measurements of secondary electron emission (SEE) induced by electron and ion bombardment on porous silicon (PS). We found that electron induced emission is strongly reduced by ion bombardment, and that this reduction is reversible. The reduction effect is large even for ion fluxes much lower compared to that of the electron beam. We attribute this effect to changes in the charge distribution of the surface dipole originated in the difference between ion and electron charge deposition depths. The nanostructure of PS plays an important role in this effect as well as in the reversibility of the process. We think that this effect could be useful in the dynamic centering and monitoring of ion and electron beams in electron spectroscopy.
\end{abstract}

The discovery of the efficient luminescence of porous silicon (PS) by Canham [1] was the starting point for a wide interest and extensive studies devoted to this material. The tailoring of PS in the range of microns to nanometers confers a peculiar behavior to this material, where quantum effects play an important role. Arrays of field emitters made of $n-$ type porous silicon show an enhanced electron emission [2]. PS diodes can operate as surface-emitting cold cathodes [3]. On the other hand, plasma generation inside the pores [4], turns PS into a base material for flat-panel field emission [5] and plasma displays. Although there are several studies about the physics of SEE dielectric films, size effects on the electronic properties of nanoporous silicon (NPSi) may introduce new and exciting peculiarities. The study of secondary electron emission (SEE) under ion bombardment is then of interest, from both basic physics and technological points of view.

The penetration depth (range) of electrons in NPSi is large than in bulk crystalline Si due to the high porosity of this material. On the other hand, since the range of ions is comparable to the characteristic dimensions of the NPSi, i.e. quite smaller than electron ranges, the density that the ions see is essentially the same as that of the bulk materials. The reason is that within these structures the atoms remain in their original crystalline sites. Thus, the natural differences among electron and ion ranges, for similar kinetic energies, are increased due to the porous nature of NPSi. These differences promote the enhancement of the dipole moment generated by electron beam induced SEE near the surface [6] when the ion beam impinges on the surface. On the other hand, in the presence of potential neutralization mechanisms, like Auger effect [7], positive charge is also released at the very surface. This dipole moment reaches a stationary value as a result of the balance between near surface electron

\footnotetext{
*Address correspondence to this author at the INTEC, CONICET - UNL Güemes 3450 - 3000 Santa Fe, Argentina; Tel: +54 342 4559174; Fax: +54 342 4550944; E-mail: rkoro@intec.ceride.gov.ar
}

depletion due to SEE, charge coming from the electron and ion beams, and relaxation by recombination through charge transport along the tortuous paths imposed by the PS nanostructure. Field assisted SEE in insulators involves electron-hole pair creation, and, in some cases carrier ballistic flight, field-drift transport and recombination processes [8]. In nanostructure materials like porous silicon, the scattering reduction may make the electronic mean free path larger than the size of structural units. Therefore, ballistic transport may be more probable along relatively large regions of the material. Malter was the first one in describing such a process for electrolytically oxidized porous aluminum [9].

In this work, we show that a reversible reduction of the SEE induced by simultaneous ion and electron bombardment is present in nanostructure porous silicon. It is absent in mesoporous and macroporous silicon samples. We consider that this reversible ion-induced effect is related to changes in dynamically sustained dipole moment resulting from bombardment of particles with different deposition lengths and charges. The reversible character of the ion-induced effect makes it interesting for use in ion beam centering processes in FIB systems and electron spectroscopy depth analysis.

The photographs of Fig. (1) show the image produced in the display of a SEM included in an AES spectrometer when the ion beam is turned on and off. The secondary current image was electronically inverted so, that points with lower SEE look brighter. We took the photographs before, during, and after the ion bombardment and also while making the ion beam raster along different directions. As shown in the figure, the effect can be used as a monitor for ion beam centring and focusing purposes. This procedure usually involves the observation of secondary electron irreversible changes [10] induced by the ion beam, or the use of a Faraday cup. Since those procedures tend to be cumbersome tasks, the use of NPSi as a monitor for positioning the ion beam may be a good option. 


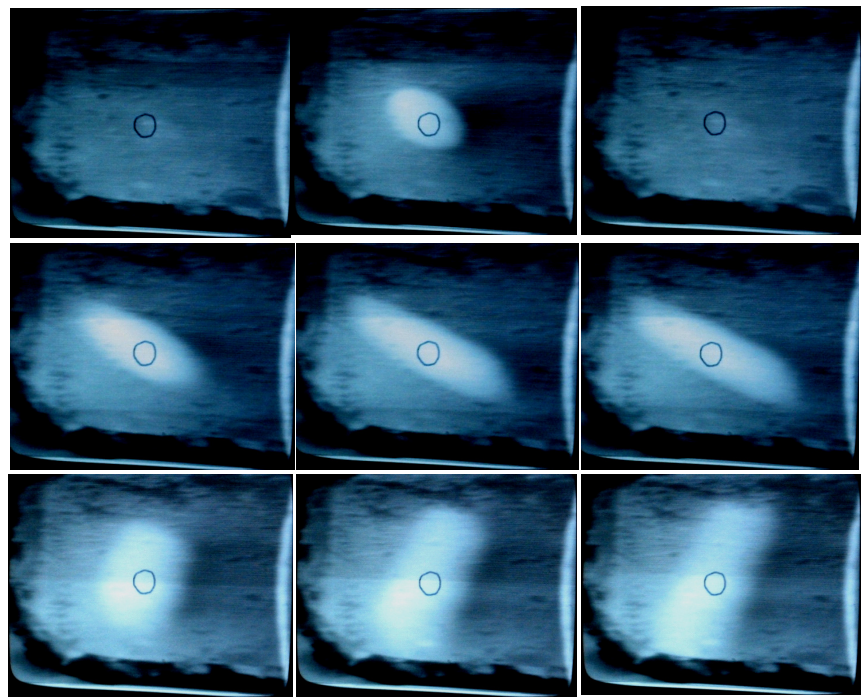

Fig. (1). SEM images of a nanoporous silicon sample (secondary electron mode). Upper row from left to right: before, during and after ion beam exposure. Middle and lower rows: during different steps of rastering along different directions. The image was electronically inverted, so that dark regions correspond to higher secondary electron emission than brighter ones.

We prepared NPSi samples by anodic etching of either $\mathrm{p}$ type $(7-17 \Omega \mathrm{cm})$ or n-type $(1-10 \Omega \mathrm{cm})$ crystalline silicon substrates using a 1:2 HF (50\%): ETOH mixture, and a current density of $20 \mathrm{~mA} / \mathrm{cm}^{2}$ for all the samples. The p-type samples were etched in darkness and the n-type ones were etched under illumination using a dichroic lamp. The intensity was around $140 \mathrm{~mW} / \mathrm{cm}^{2}$. We obtained Macroporous silicon from p-type silicon (7-17 $\Omega \mathrm{cm})$ using a 1:9 HF (50\%): Dimethylformamide mixture and a current density of $3 \mathrm{~mA} / \mathrm{cm}^{2}$. For Mesoporous silicon we used p-type silicon $(1-4 \mathrm{~m} \Omega \mathrm{cm})$ with the same electrolyte and current density as for nanoporous samples. In all the cases the samples were rinsed with ethyl alcohol after preparation and dried under $\mathrm{N}_{2}$ flow. We estimated the thickness of samples around $1 \mu \mathrm{m}$ for thin samples, and $10-30 \mu \mathrm{m}$ for thicker ones. We measured the porosity of the nanoporous samples

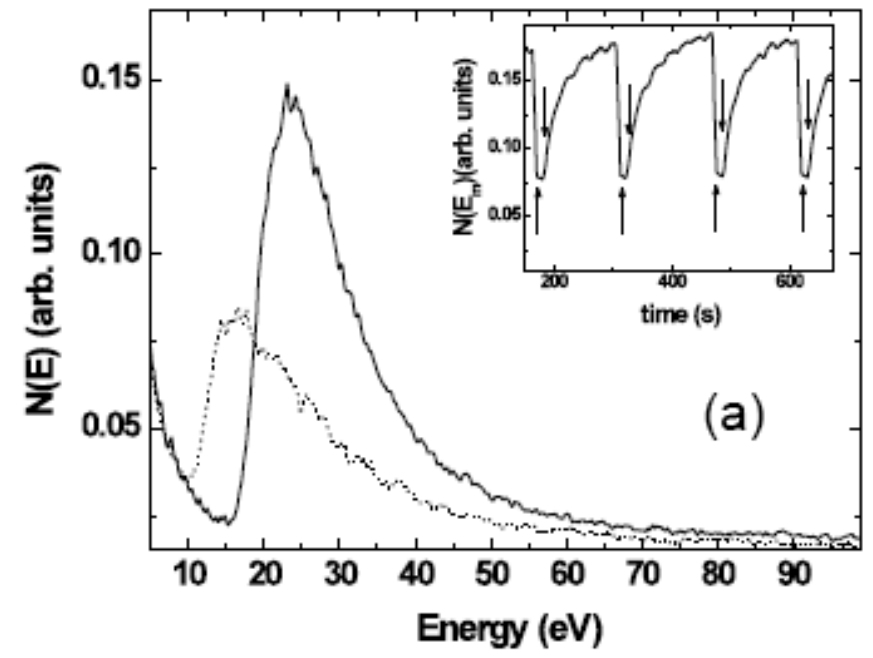

by a gravimetric method. The resulting porosity of these samples was 0.87 . We estimated the porosity of mesoporous samples as 0.55 , by fitting the reflectance spectra taken from thin layers, using the Looyenga-Landau [11] approximation.

For measuring the SEE spectra, we used a CMA based commercial Auger electron spectrometer, working with a base pressure of around $2 \times 10^{-10}$ Torr, reaching $1 \times 10^{-9}$ Torr during ion gun operation. The best conditions to acquire the electron spectra were $90 \mathrm{nA}, 400 \mathrm{eV}$ primary electron beam bombardment, rastering over a $0.4 \times 0.3 \mathrm{~mm}^{2}$ area. In order to achieve full SEE spectra we needed to polarize the samples with $-6 \mathrm{~V}$. We took the spectra alternatively under only electron bombardment, and under both, electron and $\mathrm{Ar}^{+}$ion irradiation. For ion bombardment we used $4 \mathrm{keV}$ and $9 \mathrm{nA}$ for energy and ion beam current respectively. The ion beam spot had a circular area of around $1 \mathrm{~mm}^{2}$. Within these conditions, the ion induced SEE was negligible compared to the electron one.

In Fig. (2) we show the SEE spectra for a $\sim 30 \mu \mathrm{m}$ thick oxidized n-type nanoporous sample (a) and a mesoporous one (b), exposed to only e-beam and to e-beam plus ions. We observed a strong signal decrease and an energy shift of the SEE spectrum when turning the ion bean on. The inset of Fig. (2) shows the time evolution of the SEE signal at a fixed energy, corresponding to the maximum of the electron induced SEE spectrum at the steady state. The up (down) arrow shows the turning on (off) of the ion beam. The full recovery to the initial yield after turning off the ion beam clearly shows the reversibility of the SEE reduction effect. The effect is qualitatively similar for non-intentionally oxidized n-type NPSi (not shown), and it is absolutely absent for mesoporous (Fig. 2b and inset), macroporous (not shown) and clean crystalline silicon (not shown) samples. The charging effect, responsible for the ion induced energy shift of the spectra shown in Fig. (2), turns to be negligible when using thinner $(\sim 1 \mu \mathrm{m}$ in thickness) samples.

In Fig. (3a) we show the evolution of the maximum of the SEE spectrum of the thinner sample when turning off the ion beam, for different electron beam currents. Both, the

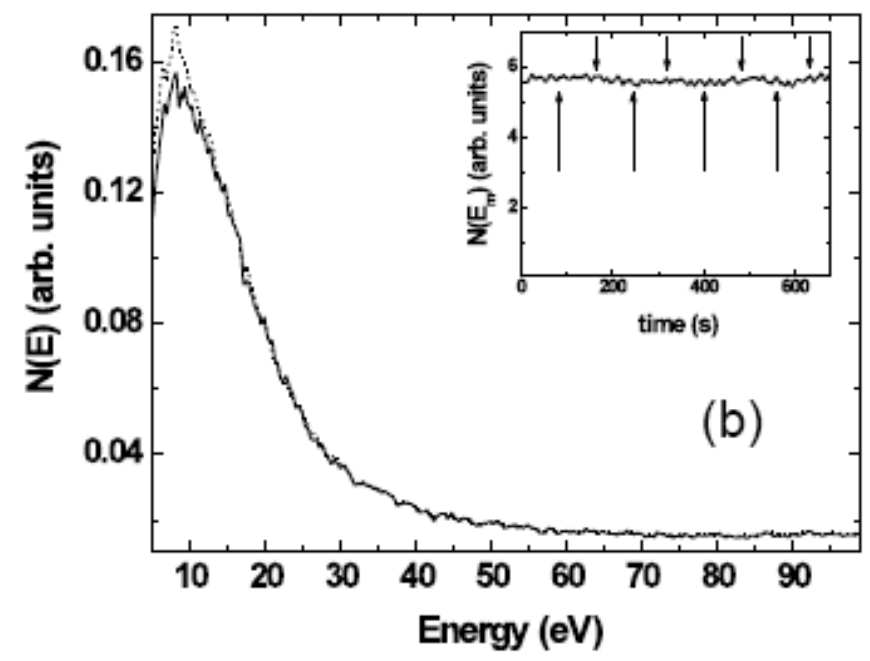

Fig. (2). Secondary electron emission spectra of a thick nanoporous silicon sample (a), and a mesoporous one (b). Full lines for electron beam induced secondary electron emission, and dotted lines correspond to the spectra taken using both the electron and the ion beams. The insets are the evolution of the maximum signal, arrows up (down) indicates when we switched ion gun on (off). 
initial (ion beam on) and the final (ion beam off) values of SEE yields depend on the electron beam current. The curves are quite well fitted with the function:

$$
I_{S}=I_{o}[1-A \cdot \exp (-t / \tau)]
$$

We propose an explanation of the whole behavior in the basis of a capacitive effect. No matter the type of bombardment particle, secondary electron emission creates a positively charged layer close to the surface, i.e. the surface layer became depleted of electrons. Even when only the primary e-beam is operating, a positive surface layer, originated in the SEE induced electron depletion, and a negative charge, cumulated at a depth in the order of the impinging electron range, create an internal dipole [6] as shown in the scheme of Fig. (3b). On the other hand, the primary e-beam deposits the whole energy in the region in between these layers, creating electron-hole pairs, which enlarges the electrical conductivity in the zone. The charge distribution then reaches a steady state as a result of the balance between the e-h generation, trapping processes, and carrier drift induced by the internal field of the dipole. An additional charge relaxation effect occurs between the implanted negative region and the crystalline silicon substrate. This relaxation takes place slowly due to the large PS resistivity originated in the quantum confinement enlarging of the gap [12]. In summary, a steady state dipole moment results from a dynamic balance between charging and relaxation processes. Within this picture, when the ion beam strikes the sample, the positive surface charge increases due to the very surface nature of ion interactions, enhancing the value of the internal dipole moment. Since the electric field near the positive surface layer precludes the electron emission, the SEE signal should decay, as it actually occurs (Fig. 2a). When we turned the ion beam off, the implanted charge exponentially decays [6] so the SEE emission should recover the only e-beam irradiation value, following the same rule, shown in Fig. (3). Finally,

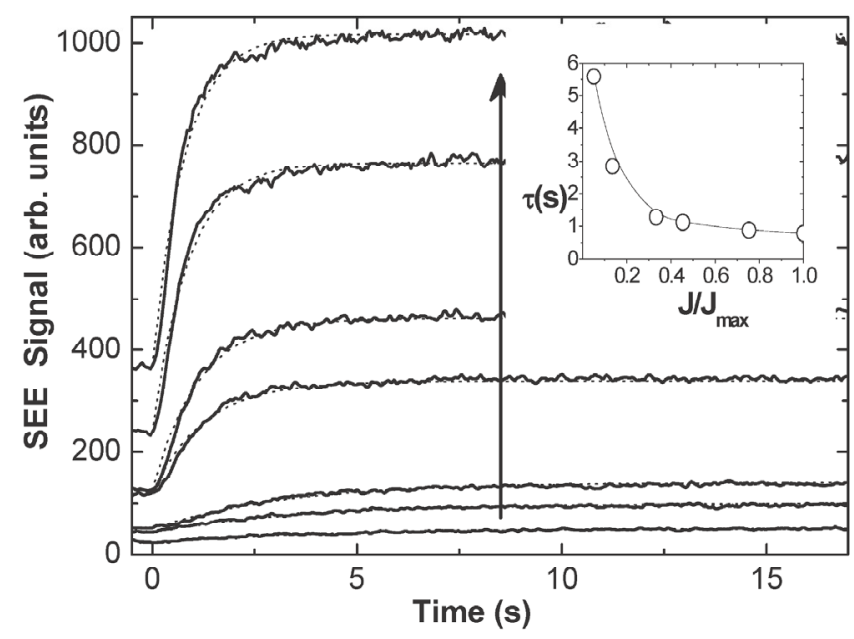

(a) considering the surface dipole as a capacitor, and taking into account that the greater the primary e-beam current the greater the rate of e-h pairs generation and the greater the conductivity of the irradiated layer, the relaxation of the charge should follow an exponential decaying with a time constant proportional to the inverse of the conductivity. Thus, the model predicts a decrease of $\tau$ with e beam intensity, as we depict in the inset of Fig. (3).

The mesoporous and the macroporous samples have larger conductivities than NPSi, allowing a faster charge rearrangement, and making possible a faster relaxation of the surface dipole. Moreover, since the carrier density is large, screening effects are more important than in lower conductivity materials like NPSi, so that the width of the positive space charge region is lower. As a consequence the ion induced surface charge changes are negligible for these materials, conspiring once more against the formation of the surface dipole. The vanishing of ion induced changes in the retarding field predicts, within our model, that the ion induced SEE reduction should operate for nano but not for meso and macroporous samples as it actually occurs (Figs. 2a, b). Additional evidence provided by measurements of the effect performed over increasingly oxidized NPSi films (not shown) supports the idea that the magnitude of the effect is lower for more conductive samples.

On the other hand, due to the fractal nature of the PS nanostructure, the characteristics of the surface remain essentially unchanged under ion bombardment. Thus, there are no permanent changes in the SEE after we turned the ion beam off. The reversibility of the ion induced SEE reduction effect reported in the present work can be useful in the development of ion focusing and centering devices [13], (see photo shown in Fig. (1)).

In summary:

1. We found that nanoporous silicon presents an electron induced SEE reduction under ion

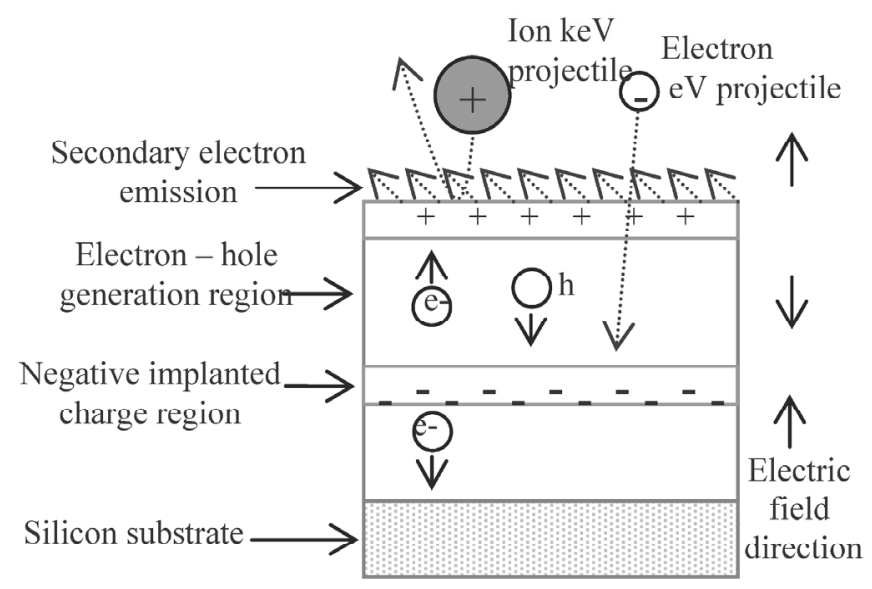

(b)

Fig. (3). (a) Full lines: evolution of the secondary electron emission signal for different currents of the primary e-beam, after turning off the ion gun. Dot lines: fitting using Eq. (1). The arrow indicates the sense of increase of the electron beam current densities in the inset, $\mathrm{J} / \mathrm{Jmax}$ is the normalized saturation current, and $\tau$ is defined in Eq. (1). (b) Schematic picture of the charging process during e-beam (and also ion beam) exposure. 
bombardment, which is clearly observable through scanning secondary electron microscopy.

2. The SEE reduction effect can be explained through a ballistic electron interaction with a surface dipolar electric field. The dipole is originated in the different penetration range of electrons and ions and the structure of porous silicon.

3. Based on this effect we have developed a system to monitor the shape and position of an ion beam.

\section{ACKNOWLEDGEMENTS}

We are indebted to Dr. John Weisz for the kind revision of this paper. We also wish to acknowledge financial support from CAI+D 002-011 (2005) (UNL), PIP 5730 / 5277 (CONICET), and PICTs 05 32515/ 061138 (SECyT).

\section{REFERENCES}

[1] Canham LT. Silicon quantum wire array fabrication by electrochemical and chemical dissolution of wafers. Appl Phys Lett 1990; 57: 1046-48.

[2] Takai M, Yamashita M, Wille H, et al. Enhanced electron emission from n-type porous Si field emitter arrays. Appl Phys Lett 1995; 66: 422 .
[3] Koshida N, Sheng X, Komoda T. Quasiballistic electron emission from porous silicon diodes. Appl Surf Sci 1999; 146 (1): 371- 6.

[4] Tam HL, Kung EW, Zhang XX, Gong ML, Cheah KW. Generation of plasma emission from porous silicon. In: Optoelectronics. IEEE: Proceedings of the Sixth Chinese Symposium; Sept 2003; IEEE, New Jersey, Ltd, United States of America 2003; DOI 10.1109/ COS.2003.1278180.

[5] Kleps I, Nicolaescu D, Lungu C, Musa G, Bostan C, Caccavale F. Porous silicon field emitters for display applications. Appl Surf Sci 1997; 111: 228- 32 .

[6] Thiel BL, Toth M. Secondary electron contrast in low-vacuum environmental scanning electron microscopy of dielectrics. J Appl Phys 2005; 97(5): 1-18.

[7] Hagstrum HD. Auger ejection of electrons from tungsten by noble gas ions. Phys Rev 1954; 96: 325-35.

[8] Fitting HJ, Cornet N, Touzin M, Goeuriot D, Guerret-Piécourt C, Tréheux D. Injection of charge nanostructures into insulators. J Eur Ceram Soc 2007; 27 (13-15): 3977- 82.

[9] Malter L. Thin film field emission. Phys Rev 1936; 50: 48-58.

[10] Tseng F, Chen M, Chen A, Chen H. Method for sectioning a semiconductor wafer with FIB for viewing with SEM. US20016252227, 2001.

[11] Theiss W. Optical properties of porous silicon. Surf Sci Rep 1997; 29: 91-192.

[12] Koropecki RR, Arce RD, Schmidt JA. Photo-oxidation effects in porous silicon luminescence. Phys Rev B 2004; 69: 1-6.

[13] Koropecki RR, Ferron J, Ruano Sandoval GD. Procedimiento para el monitoreo y centrado de uno o más haces de partículas energéticas. Patent Pending, P 090100486, February 12, 2009.

This is an open access article licensed under the terms of the Creative Commons Attribution Non-Commercial License (http://creativecommons.org/licenses/by-nc/ $3.0 /$ ) which permits unrestricted, non-commercial use, distribution and reproduction in any medium, provided the work is properly cited. 